\title{
8 Point DFT Computation Using Butterfly Structure on Vertex KIT
}

\author{
Sonakshi Halli, M. Roopa, M. Rajeshwari, M. Suhasini and Channakka Lakkannavar
}

\begin{abstract}
The paper carried out describes the process of exploring the Chip scope Pro VIO through the Xilinx platform and implementing the 8 DFT computation using the butterfly structure and analyzing the same. The project also depicts the steps to be carried out in the analyzing process. The report includes the methodology used for designing, analyzing and verification, simulation of the result. The simulation is one of the extensively used method for automotive and other real time processes. The Chip scope Pro $\mathrm{VIO}$ is also one of such method used.
\end{abstract}

Keywords-- Vertex, FFT ( Fast Fourier Transform)

\section{INTRODUCTION}

$\mathrm{T}$ HE Fast Fourier Transform Algorithm plays an important role in digital signal processing. FFT algorithms are basically Fast Fourier Transform algorithms. They are used to calculate DFT efficiently. Because of FFT algorithms DFT can be calculated in real time. DSP processors have special architectural provisions to implement FFT algorithms efficiently. Several algorithms have been developed in order to reduce the computational complexity such as Radix-2, Radix4, Radix-8, Split radix method. These algorithms are either based on decimation in time or decimation in frequency.

The radix-2 FFT algorithms are based on divide and conquer approach. In this approach the $\mathrm{N}$-point DFT successfully decomposed into smaller DFTs. Because of this decomposition, the number of computations are reduced. Let value of ' $N$ ' be selected such that $N=2^{v}$. This $N$-point DFT is decomposed successively such that smallest DFT will be of size $\mathrm{N}=2$. Hence this type of algorithms are called as Radix-2, or radix of these algorithms is ' 2 '. The basic building block of FFT algorithms is Butterfly structure.

\section{METHODOLOGY}

In this section we present several methods for computing the DFT efficiently. In view of the importance of the DFT in various digital signal processing applications, such as linear filtering, correlation analysis, and spectrum analysis, its efficient computation is a topic that has received considerable

Sonakshi Halli, Department of Electronics and Communication, SDM College of Engineering and Technology.

M. Roopa, Department of Electronics and Communication, SDM College of Engineering and Technology.

M. Rajeshwari, Department of Electronics and Communication, SDM College of Engineering and Technology.

M. Suhasini, Department of Electronics and Communication, SDM College of Engineering and Technology.

Channakka Lakkannavar, Department of Electronics and Communication, SDM College of Engineering and Technology. DOI:10.9756/BIJRCE.8211 attention by many mathematicians, engineers, and applied scientists. From this point, we change the notation that $X(k)$, instead of $y(k)$ in previous sections, represents the Fourier coefficients of $x(n)$.

Basically, the computational problem for the DFT is to compute the sequence $\{\mathrm{X}(\mathrm{k})\}$ of $\mathrm{N}$ complex-valued numbers given another sequence of data $\{\mathrm{x}(\mathrm{n})\}$ of length $\mathrm{N}$, according to the formula

$$
\begin{gathered}
X(k)=\sum_{n=0}^{N-1} x(n) W_{N}^{k n}, \quad 0 \leq k \leq N-1 \\
W_{N}=e^{-j 2 \pi / N}
\end{gathered}
$$

In general, the data sequence $x(n)$ is also assumed to be complex valued.

Similarly, the IDFT becomes,

$$
x(n)=\frac{1}{N} \sum_{n=0}^{N-1} X(k) W_{N}^{-n k}, \quad 0 \leq n \leq N-1
$$

Basically DFT and IDFT involve the same type of computations. In particular, there are two properties for twiddle factor:

$$
\begin{aligned}
& \text { Symmetry property: } \mathrm{W}_{\mathrm{N}}{ }^{\mathrm{k}+\mathrm{N} / 2}=-\mathrm{W}_{\mathrm{N}}{ }^{\mathrm{k}} \\
& \text { Periodicity property: } \mathrm{W}_{\mathrm{N}}{ }^{\mathrm{k}+\mathrm{N}}=\mathrm{W}_{\mathrm{N}}{ }^{\mathrm{k}}
\end{aligned}
$$

Symmetry and periodicity properties of the twiddle factor does not hold good for DFT. So, DFT becomes inefficient. The efficient algorithms collectively known as FFT algorithms, exploit these two basic properties of the twiddle factor. Figure 1 shows the computation of $\mathrm{N}=8$ point DFT. There are three stages in computation of 8 point DFT. In the first stage four 2 point DFTs, in the second stage two 4 point DFTs and in third stage one 8 point DFT are computed.

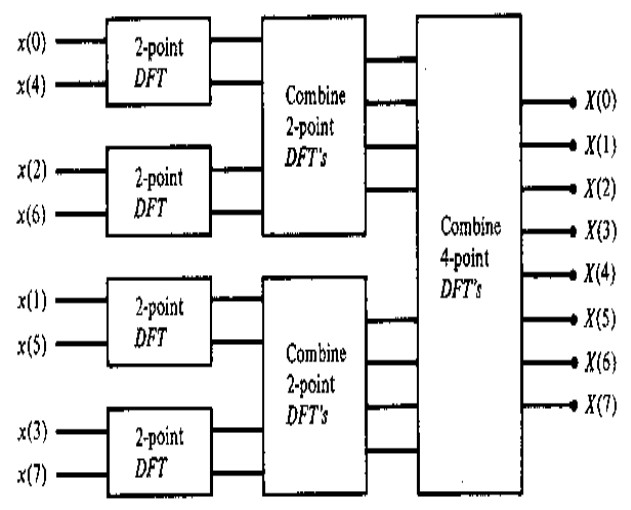

Figure 1: Stages in the 8 Point DFT Computation 


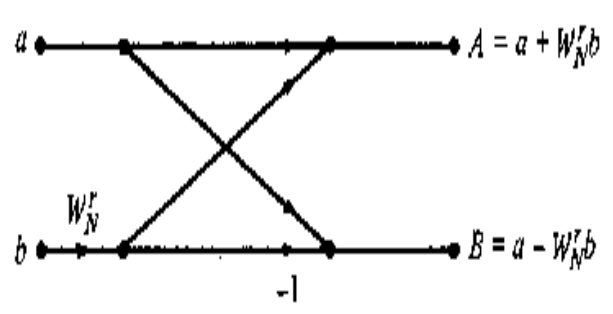

Figure 2: Butterfly Structure

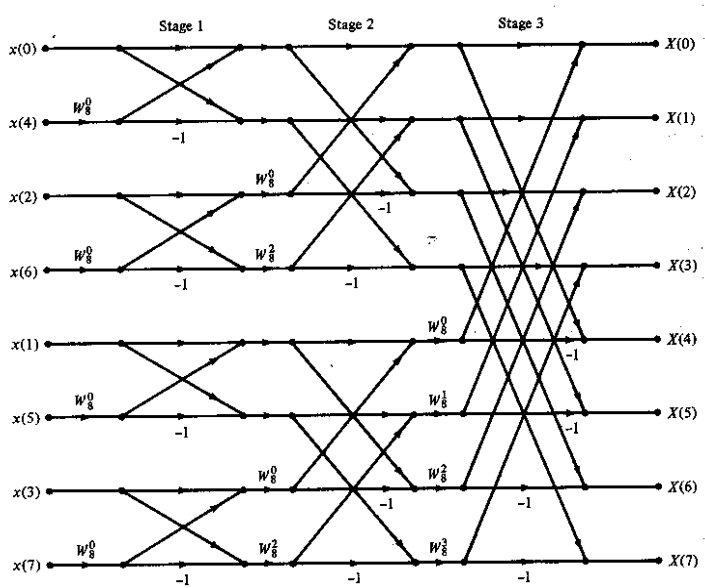

Figure 3: Flow Diagram of 8 Point DFT

For $N=8, x(0), x(2), x(4), x(6), x(1), x(1), x(3), x(5), x(7)$ is the sequence after the first decimation and $x(0), x(4), x(2)$, $x(6), x(1), x(5), x(3), x(7)$ is the sequence after the second decimation. Figure 4 shows the decimation of 8 point sequence.

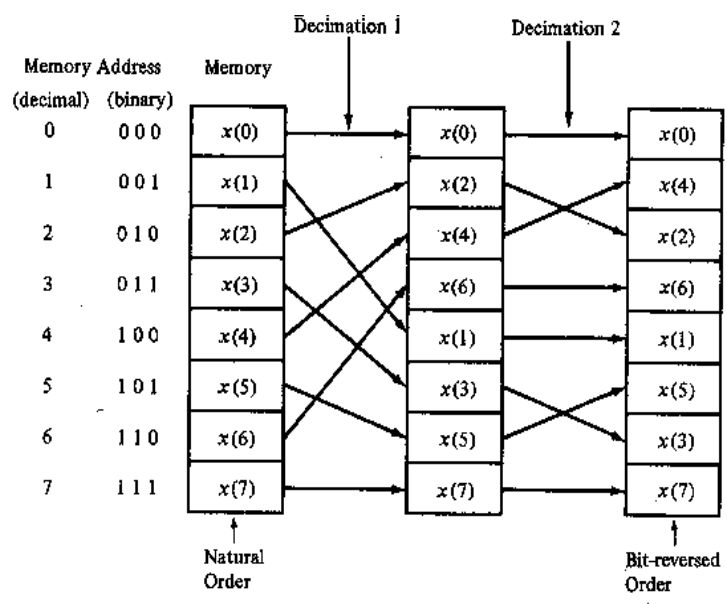

(a)

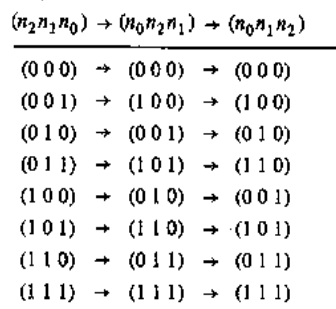

(b)

Figure 4: Decimation of 8 Point Sequence

\section{IMPLEMENTATION OF 8 POINT DFT IN HARDWARE}

The 8 point DFT implemented here consists of 16 inputs which include real parts of 64 bits, clock of 1 bit, select line of 3 bits and 2 outputs which includes real and imaginary parts of 16 bits. Hence total number of input and output bits are 68 and 16 respectively.

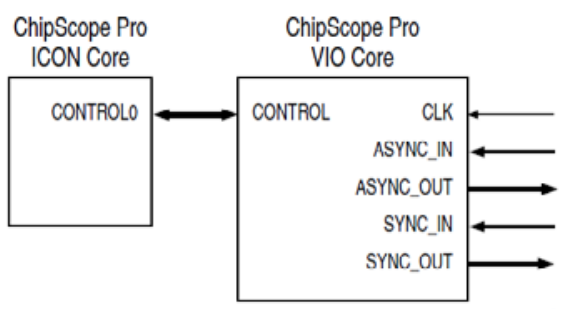

Figure 5: VIO and ICON Communication

\section{A. Core Generator}

After entering the VIO core parameters, click Generate to create the VIO core files. After the VIO core has been generated, a list of files that are generated will appear in a separate window called "Readme < corename $>$ ".

The CORE ${ }^{[4]}$ Generator tool provides the ability to define and generate a customized VIO core for adding virtual inputs and outputs to our HDL designs. We can customize the virtual inputs and outputs to be synchronous to a particular clock in our design or to be completely asynchronous with respect to any clock domain in our design. We can also customize the number of input and output signals used by the VIO core.

\section{B. Using the VIO Core}

To instantiate the example VIO core HDL files into our design, use the following guidelines to connect the VIO core port signals to various signals in our design:

1. Connect the VIO core's CONTROL port signal to an unused control port of the ICON core instance in the design.

2. Connect all unused bits of the VIO core's asynchronous and synchronous input signals to a "0". This prevents the mapper from removing the unused trigger and/or data signals and also avoids any DRC errors during the implementation process.

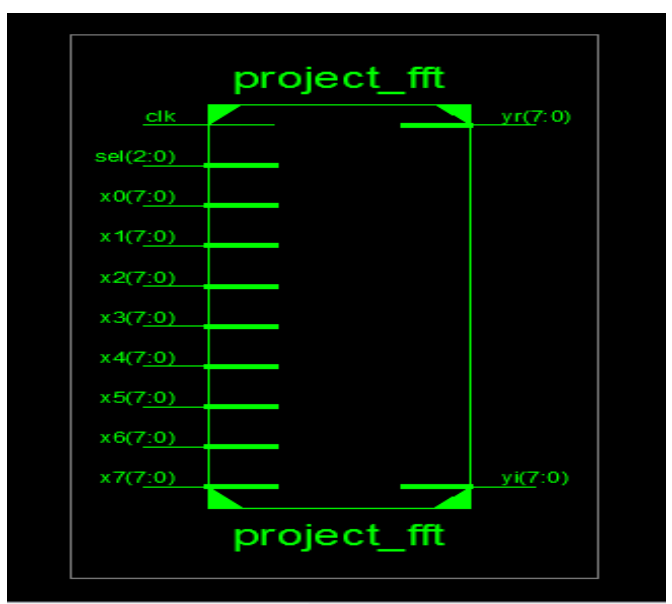

Figure 6: Top Level Module of 8 Point DFT 


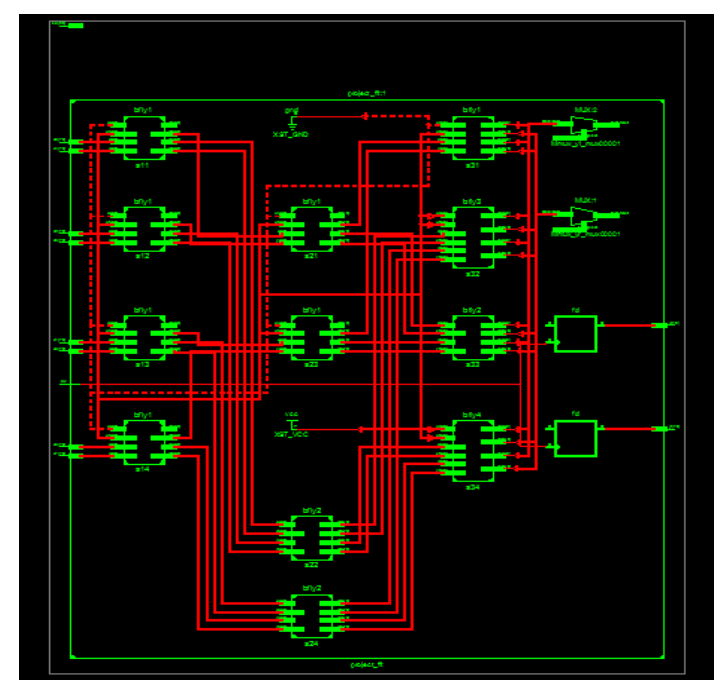

Figure 7: RTL Schematic of 8 Point DFT

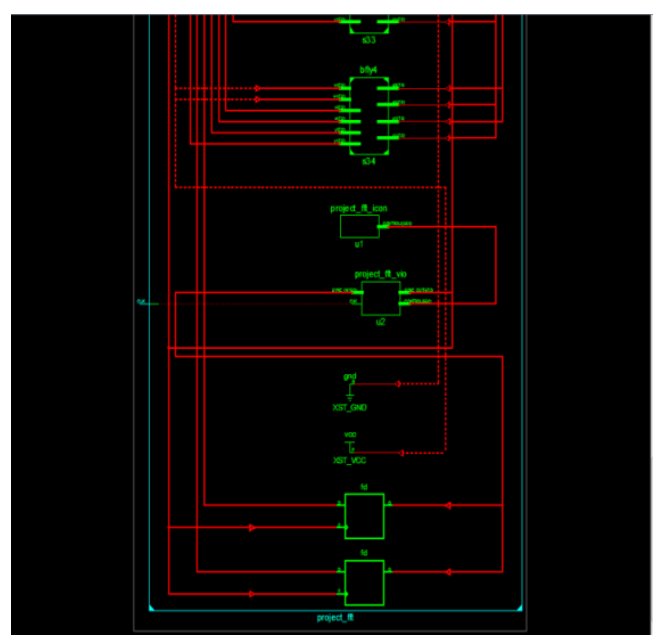

Figure 8: Block Diagram of Interconnection between VIO, ICON to FFT Block

\section{Results Through VIO AND CHIPSCOPE Pro}

The output on the console window of the Chipscope $\mathrm{Pro}^{[3]}$ is obtained by changing the inputs manually and the output changes as follows.

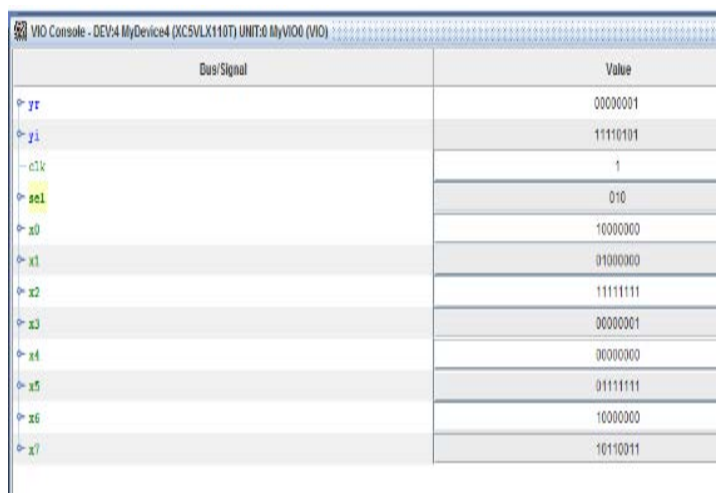

Figure 9: VIO Console Window

\section{DESIGN SUMMARY}

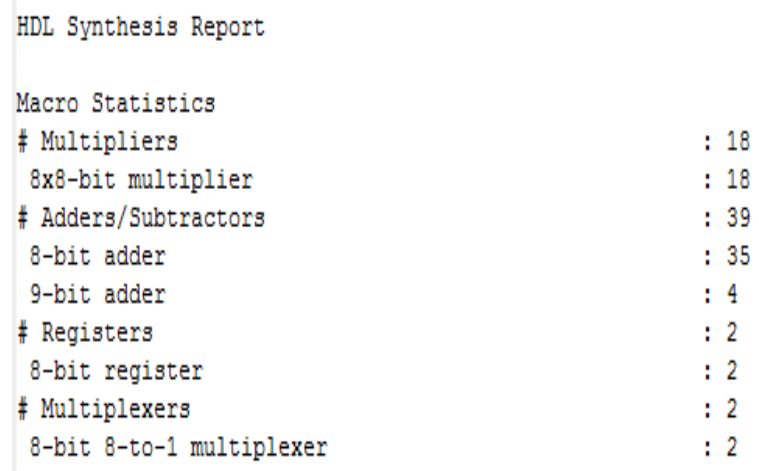

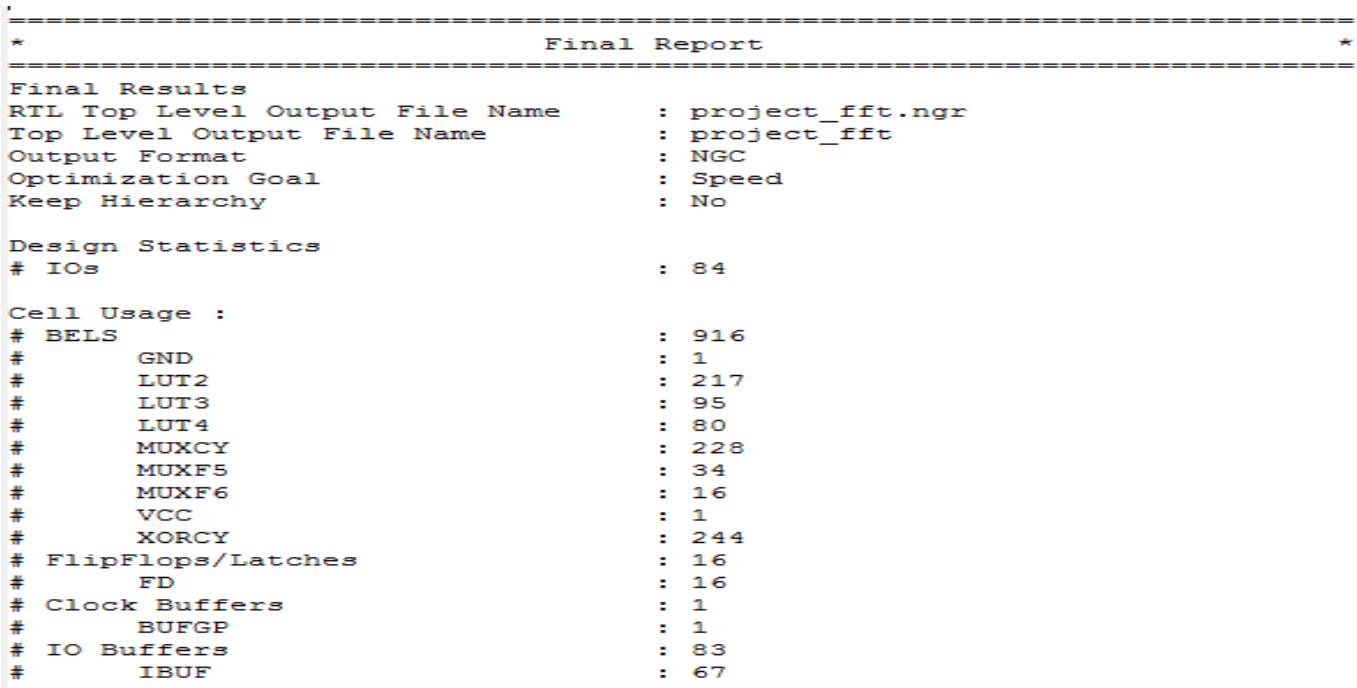

\section{CONCLUSION}

This paper shows how Chipscope pro VIO core is customizable core. The design steps are verified in Xilinx ISE Design Suite 14.5 and then through the Reconfigurable
Device.

The cost effective and efficient DFT computation is modeled and implemented using the Chipscope Pro and VIO Console. 


\section{REFERENCES}

[1] J.W. Cooley and J.W. Tukey, "An algorithm for the machine calculation of complex Fourier series”, Mathematics of computation, Vol.19, No.90, Pp.297-301, 1965.

[2] P. Duhamel and M. Vetterli, "Fast Fourier transforms: a tutorial review and a state of the art”, Signal processing, Vol.19, No.4, Pp.259-299, 1990.

[3] DS284, Xilinx, “ChipScope Pro VIO, vol.1.00a, 1.01a, 1.02a, 1.03a”, 2009.

[4] DS284, Xilinx, "LogiCORE IP ChipScope Pro Virtual Input/output, (VIO) (1.04a)", 2011.

[5] F.M. Vallina, C. Kohn and P. Joshi, "Zynq all programmable SoC Sobel filter implementation using the Vivado HLS tool”, Application Note XAPP890, Xilinx, 2012.

[6] G.P. John and G.M. Dimitris, "Digital Signal Processing: Principles, Algorithms and Applications”, Pentice Hall, 1996.

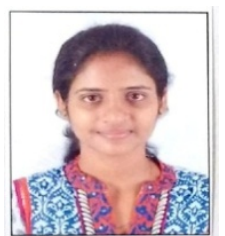

\section{AUTHOR's PROFILE}

Sonakshi Halli is a final year undergraduate student, Dept. of Electronic and Communication Engineering from SDM College of Engineering and Technology, Dharwad, Karnataka. Main area of interest are C, C++ Programming, Analog Electronics.

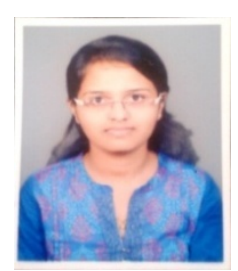

Roopa Mahantannavar is a final year undergraduate student, Dept. of Electronic and Communication Engineering from SDM College of Engineering and Technology, Dharwad, Karnataka. Main area of interest are Digital Circuit Design, C and C++ Programming.

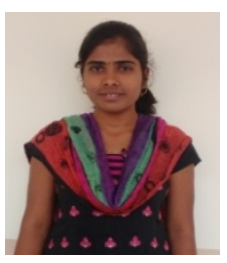

Rajeshwari Managuli is a final year undergraduate student, Dept. of Electronic and Communication Engineering from SDM College of Engineering and Technology, Dharwad, Karnataka. Main area of interest are Analog Electronics and Automotive Electronics, Digital Circuit Design.

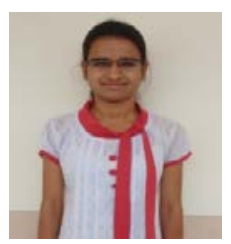

Suhasini Madannavar is a final year undergraduate student, Dept. of Electronic and Communication Engineering from SDM College of Engineering and Technology, Dharwad, Karnataka. Main area of interest are Digital Circuit Design and Analog Electronics. 